\title{
3 Research Square

\section{The miR528-D3 module regulates plant height in rice by modulating the gibberellin and abscisic acid metabolisms}

\section{Xing Liu}

Zhejiang A\&F University

Juan Zhao

Zhejiang A\&F University

Mei Wang

Zhejiang A\&F University

Lingjuan Xie

Zhejiang A\&F University

\section{Zhengxin Wu}

Zhejiang A\&F University

Jiuming Yu

Zhejiang A\&F University

Yuchen Wang

Zhejiang A\&F University

Zhiqiao Zhang

Zhejiang A\&F University

Yufang Jia

Zhejiang A\&F University

Qingpo Liu ( $\square$ liuqp@zafu.edu.cn )

Zhejiang A\&F University https://orcid.org/0000-0002-6249-5796

\section{Research Article}

Keywords: Rice, dwarf, plant height, gibberellin, abscisic acid

Posted Date: August 25th, 2021

DOI: https://doi.org/10.21203/rs.3.rs-812323/v1

License: (c) (1) This work is licensed under a Creative Commons Attribution 4.0 International License.

Read Full License 


\section{Abstract \\ Background}

Plant height, as one of the important agronomic traits of rice, is closely related to yield. In recent years, plant height-related genes have been characterized and identified, among which the $D 3$ gene is one of the target genes of miR528, and regulates rice plant height and tillering mainly by affecting strigolactone (SL) signal transduction. However, it remains unknown whether the miR528 and D3 interaction functions in controlling plant height, and the underlying regulatory mechanism in rice.

\section{Results}

In this study, we found that the plant height, internode length, and cell length of internodes of $d 3$ mutants and miR528-overexpressing (OE-miR528) lines were greatly shorter than WT, D3-overexpressing (OE-D3), and miR528 target mimicry (OE-MIM528) transgenic plants. Knockout of D3 gene (d3 mutants) or miR528-overexpressing (OE-miR528) triggers a substantial reduction of gibberellin (GA) content, but a significant increase of abscisic acid (ABA) accumulation than in WT. The $d 3$ and OE-miR528 transgenic plants were much more sensitive to GA, but less sensitive to ABA than WT. Moreover, the expression level of GA biosynthesis-related key genes, including OsCPS1, OsCPS2, OsKO2 and OSKAO was remarkably higher in OE-D3 plants, while the NECD2 expression, a key gene involved in ABA biosynthesis, was significantly higher in $d 3$ mutants than in WT and OE-D3 plants.

\section{Conclusion}

The results indicate that the miR528-D3 module negatively regulates plant height in rice by modulating the GA and ABA homeostasis, thereby further affecting the elongation of internodes, and resulting in lower plant height, which adds a new regulatory role to the $D 3$-mediated plant height controlling in rice.

\section{Background}

As one of the important components of plant architecture, plant height is closely related to the photosynthetic efficiency and lodging resistance, and directly affects the biomass of plants in rice (Zhang et al. 2014). Accordingly, appropriate dwarfing of plant height will benefit in improving the lodging resistance of rice. In the 1950s, the discovery and application of the rice semi-dwarf gene $s d 1$ greatly alleviate the food shortages in at least nineteen developing countries (Monna et al. 2002; Wang and Wang 2017). It is thus feasible that genetically manipulating of functional genes should be effective in controlling plant height and further grain yield in rice.

In recent years, amounts of genes related to plant height have been characterized and identified in rice, where most of them are involved in plant hormone metabolism and signal transduction, such as 
gibberellin (GA), brassinolide (BR), strigolactone (SL) and abscisic acid (ABA) (Zou et al. 2019; Zhang et al. 2020a). The OsKO2 gene encodes a kaurene oxidase that catalyzes the early steps of GA biosynthesis. The expression of OsKO2 was repressed by LRK1 and activated by OsbZIP58, thereby affecting GA biosynthesis and internode elongation in rice (Yang et al. 2013; Wu et al. 2014). GID1 encodes a soluble GA signal receptor, which mediates GA signal transduction and plays a major role in regulation of rice plant height (Ueguchi-Tanaka et al. 2005). Except for GA, BRs are also determinant in controlling plant height. The plant height of BR-deficient dwarf mutant brd 2 was about $70 \%$ of the wild type (WT) at the seedling stage, but the mutants were severe dwarfing, only $40 \%$ of the WT plants, and most of the internodes did not elongate except the uppermost internode at the mature stage (Hong et al. 2005). In addition, D2, D11/CYP724B1, D61/OsBR/1, OsDWARF4 also control plant height by regulating BR synthesis and signaling (Zhang et al. 2016; Tong et al. 2018). Interestingly, previous studies reveal that the abnormal signal pathways related to $S L$, such as $d 88 / d 14, d 27, d 3$, dit1, often cause changes in tiller number and plant height (Ishikawa et al. 2005; Arite et al. 2009; Gao et al. 2009; Lin et al. 2009). Another kind of hormone ABA negatively regulates plant growth, and results in dwarfing of plants, which often functions in the way with GA antagonism (Wang et al. 2020).

In rice, $D 3$ encodes a leucine-rich repeat F-box protein, which is homologous to MAX2/ORE9 that controls the activity of axillary buds by participating in an SCF complex and SL signal transduction in Arabidopsis thaliana (Ishikawa et al. 2005; Zhao et al. 2014; Sun et al. 2018). The rice $d 3$ mutant exhibits a shorter plant height and increased tiller numbers (Ishikawa et al. 2005; Zhao et al. 2014; Sun et al. 2018).

Recently, D3 was found to be involved in a karrikin signaling complex (D14L-D3-OsSMAX1) that mediates the regulation of rice mesocotyl elongation in the dark (Choi et al. 2020; Zheng et al. 2020). In addition, $D 3$ was experimentally validated to be a target gene of rice miR528 through degradome sequencing ( $\mathrm{Li}$ et al. 2010; Zhou et al. 2010). As known, miR528 plays multifaceted roles in regulation of plant growth and development, and biotic and abiotic stress responses (Liu et al. 2015; Wu et al. 2017; Tang and Thompson 2019; Yang et al. 2019; Zhang et al. 2020b; Wang et al. 2021). However, whether the miR528 and $D 3$ interaction plays a role in regulation of plant height in rice, and its further underlying regulatory mechanism remains unclear.

In this study, we aim to uncover the regulatory role of the miR528-D3 module in controlling plant height by regulating the expression of some key genes related to GA and ABA biosynthesis, leading to the changes of the content of endogenous hormones and the cell elongation in rice. This research is significant for further understanding the $D 3$-mediated mechanism in regulation of plant height in plants.

\section{Results}

\section{miR528 and D3 exhibit opposite expression patterns along developmental stages}

$D 3$ is reported to be one of the target gene of miR528 in rice (Li et al. 2010; Zhou et al. 2010). To explore whether the $D 3$ expression is regulated by miR528, we first investigated their temporal and spatial expression patterns in different tissues along developmental stages. It is obvious that miR528 and D3 
showed totally different expression patterns (Fig. 1). The transcriptional level of miR528 was relatively lower in roots, shoots, and leaves at the seedling and tillering stages, but was significantly higher in the examined tissues at the heading and mature stages, except for in the stems and stem nodes at the maturity stage (Fig. 1A). On the contrary, the $D 3$ gene was highly expressed in roots and leaves at the seedling stage and in roots, shoots, and leaves at the tillering stage, while its expression level was greatly lower in other tested tissues, especially in leaves at the mature stage (Fig. 1B). These observations strongly support that the expression of $D 3$ is truly controlled by miR528 in rice.

\section{The miR528-D3 module negatively regulates plant height in rice}

In order to better understand whether the miR528-D3 module have potential roles in regulation of plant height in rice, two independent D3-overexpressing transgenic lines (OE-D3-2 and OE-D3-9) and two homozygous $D 3$-knockout mutant lines (d3-1 and d3-3) were developed. The $D 3$ gene was significantly up-regulated in the OE-D3 lines, whose transcriptional level was about 12- and 166-fold higher than in WT. The d3-1 mutant had a deletion of three nucleotides (ACG) at the position of 101-103 bp in the coding region, leading to the deletion of the aspartic acid (D) at the 34th residues, while the $d 3-3$ mutant contained a deletion of four nucleotides (GACG) at the position of 100-103 bp in the coding region, resulting in a frameshift mutation (Fig. S1).

Compared with WT plants, the plant height of $d 3-3$ and $d 3-1$ decreased by $29.9 \%$ and $26.4 \%$ at the tillering stage; Consistently, the plant height of OE-miR528 lines was apparently shorter also, which decreased by $15.5 \%$ and $13.8 \%$ in OE-miR528-10 and OE-miR528-5 plants. By contrast, OE-D3 and OE-MIM528 lines were slightly higher than WT (4.4\% and 2.0\% for OE-D3-2 and OE-D3-9, and $2.1 \%$ and $2.3 \%$ for OEMIM528-1 and OE-MIM528-16) (Fig. 2A-B). At the maturity stage, the difference on plant height was more pronounced among transgenic lines. The plant height of $d 3$ mutants and OE-miR528 lines was decreased by $69.7 \%(d 3-3), 36.9 \%$ (d3-1), $19.3 \%$ (OE-miR528-10), and 10.2\% (OE-miR528-5), while the plant height of OE-D3 and OE-MIM528 lines was increased by 12.3\% (OE-D3-2), 4.3\% (OE-D3-9), 5.6\% (OE-MIM528-1), and $8.9 \%$ (OE-MIM528-16), in comparison with WT plants (Fig. 2C-D). The results indicate that miR528 and $D 3$ genes both have regulatory effect on plant height in rice, but they function in opposite ways. In addition, $d 3-3$ was more dwarfed than $d 3-1$ at the mature stage (Figs. $2 \mathrm{C}$ and $3 \mathrm{~B}$ ), which may be due to the loss-of-function mutation occurred in $d 3-3$ plants.

\section{The dwarfness of d3 and OE-miR528 plants are due to the shorter internodes and cell length}

To reveal the histological mechanism of the changes in plant height, we counted and measured the internode number and length of WT and transgenic lines at the maturity stage. There was no difference in internode numbers between WT and transgenic plants, both of them had five internodes (Fig. 3A and C). However, the internode length of $d 3$ and OE-miR528 lines were significantly shorter, while the internode length of OE-D3 and OE-MIM528 lines were slightly longer than WT (Fig. 3A and C). The uppermost internode exhibited the most prominent difference among different transgenics (Fig. 3A and C). Thus, it is reasonable to infer that the miR528-D3 module affects rice plant height by controlling the length of internodes, especially the uppermost internode. 
Previous studies show that the length of internodes is related to the stem elongation and growth caused by cell division and expansion of the apical meristem (SAM) and the intermediate meristem (IM) in rice (Wang et al. 2018). Accordingly, we speculate that the observed difference in the internode length of transgenic rice plants may be due to the changes in the size and/or number of cells. To verify this, longitudinal tissue sections were made from the uppermost internode of WT and transgenic lines at the tillering stage. The results showed that the cell length of $d 3$ and OE-miR528 lines was greatly shorter, while the cell length of OE-D3 and OE-MIM528 was significantly longer than WT (Fig. 4). But, no obvious difference in cell numbers was found. These data indicate that the elongation but not the number of cells is responsible for the length of internode.

\section{The accumulation of GA and ABA is severely affected in d3 and OE-miR528 lines}

A variety of plant hormones affect the elongation of stems in rice, of which GA deficiency or abnormal signal transduction will cause the dwarfness of plants, while ABA has antagonistic effect of GA on plant height (Ayano et al. 2015). To clarify whether the miR528-D3 module controls internode elongation by affecting the metabolism of GA and ABA, we determined the content of endogenous $\mathrm{GA}_{3}$ and ABA of WT and transgenic lines at the seedling stage. Compared with WT, $d 3-1$ and OE-miR528-10 lines had significantly lower $\mathrm{GA}_{3}$ content, whereas OE-D3-9 and OE-MIM528-1 lines contained extensively much more $\mathrm{GA}_{3}$ (Fig. 5A). The ABA content of $d 3-1$ and OE-miR528-10 lines was significantly higher than WT, but there was no significant difference in ABA content between WT, OE-D3-9, and OE-MIM528-1 lines (Fig. 5B). Thus, the dwarfness of $d 3$ and OE-miR528 plants should be attributed to the highly increased endogenous ABA content and the decreased GA content.

To further verify the responses of rice plants to GA and ABA, the WT and transgenic lines were treated with exogenous $\mathrm{GA}(1 \mu \mathrm{M})$ and $\mathrm{ABA}(3 \mu \mathrm{M})$. After $7 \mathrm{~d}$ and $14 \mathrm{~d}$ of $\mathrm{GA}_{3}$ treatment, the plant height of $d 3-1$ and OE-miR528-10 increased significantly (Fig. 6A-B), as reflected by their significantly higher relative elongation of plant height than WT, OE-D3-9, and OE-MIM528-1 lines (Fig. 6D). However, the relative elongation of plant height of OE-D3-9 and OE-MIM528-1 lines is not significantly different from WT plants (Fig. 6D). This observation indicates that the $d 3$ and OE-miR528 lines are more sensitive to external GA. Moreover, after 7-d of ABA supplementation, the relative elongation of plant height was not apparently different from WT and transgenic lines (Fig. 6C and E); But, along with the treatment time (14 d), the d3-1 and OE-miR528-10 plants grew significantly much faster than WT, while OE-D3-9 and OE-MIM528-1 lines were not obviously different from WT (Fig. 6C and E), suggesting that the $d 3$ and OE-miR528 lines were not sensitive to the exogenous application of ABA. These results indicated that the miR528-D3 module should be involved in the anabolic processes of GA and ABA.

\section{miR528- D3 regulates the expression of GA and ABA biosynthesis- and metabolism-related genes}

Do the decreased GA content and increased ABA accumulation in $d 3$ and OE-miR528 lines result from the expression changes of key genes involved in GA and ABA biosynthesis and metabolism? To verify this, we analyzed the expression level of four GA degradation (OsGA2ox1, OsGA2ox3, OsGA2ox4 and OsEUI), 
seven GA biosynthesis (OsGA20x, OsGA3ox2, OsCPS1, OsCPS2, OsKS5, OsKO2 and OsKAO), three ABA oxidation ( $A B A 80 \times 1, A B A 80 \times 2$ and $A B A 4)$, and four ABA biosynthesis-related genes (NECD1, NECD2, SDR1 and AAO3) in WT, OE-D3-9 and $d 3$-1 lines (Spielmeyer et al. 2002; ltoh et al. 2004; Oikawa et al. 2004; Sakamoto et al. 2004; Luo et al. 2006; Saika et al. 2007; Lo et al. 2008; Zhang et al. 2008; Zhu et al. 2009; Toyomasu et al. 2009; Huang et al. 2010; Feng and Zhao 2011; Bang et al. 2013; Qin et al. 2013; Chi et al. 2014; Endo et al. 2014; Tong et al. 2014; Zhang et al. 2020c). The GA degradation-related genes OsGA20x3 and OsEUI were highly expressed in $d 3-1$ and OE-D3-9 lines, respectively (Fig. 7A). The expression of four GA biosynthesis-related key genes, including OsCPS1, OsCPS2, OsKO2 and OsKAO, was significantly up-regulated in OE-D3-9 than that in WT and $d 3-1$ mutant (Fig. 7B). The expression level of the three ABA oxidation-related genes was similar in $d 3-1$ and OE-D3-9 lines (Fig. 7C). However, the ABA biosynthesis-related key gene NECD2 was significantly up-regulated in $d 3-1$ plants (Fig. 8D). These results indicate that the changes of plant height of transgenic lines would be partially due to the expression changes of some GA and ABA biosynthesis and metabolism-related genes.

\section{Discussion}

Plant height is one of the most important agronomic traits, which largely affects crop yields. During the past decades, a number of dwarf mutants have been identified in rice, of which $d 3$ is a tillering dwarf mutant caused by the mutation in an F-box protein with leucine-rich repeats (Ishikawa et al. 2005; Yan et al. 2007). In the present study, we newly generated two allelic mutants, $d 3-1$ and $d 3-3$, through CRISPR/Cas9 system (Fig. S1). Both the two homozygous mutants exhibit dwarf phenotype during the whole growth stages, which results from the abnormal elongation of internode cells (Fig. 4). But, $d 3-3$ has a much severer tillering dwarf phenotype than $d 3-1$, which may be due to the introduced loss-of-function mutation that caused its prematurely transcription termination. On the contrary, D3-overexpressing plants are relatively higher than WT (Figs. 2 and 3). Accordingly, D3 should be a crucial gene in regulation of plant height in rice.

miR528 is a monocot-specific miRNA, which plays multifunctional roles in plants (Wu et al. 2017; Tang and Thompson 2019; Yang et al. 2019; Zhang et al. 2020a). But, whether miR528 regulates plant height remains unknown. In rice, D3 is one of the target genes of miR528 with the target site located in the $3^{\prime}$ UTR (Li et al. 2010; Zhou et al. 2010; Tang and Thompson 2019). We found that the plant height of OEmiR528 transgenics was significantly lower than WT, but was greatly higher than the $d 3$ mutants (Figs. 2 and 3). These observations indicate that the rice miR528 plays an important role in regulation of plant height by targeting the $D 3$ gene. However, in OE-miR528 lines, the $D 3$ expression may maintain at a relative level, although it was substantially down-regulated by miR528, suggesting that the plant height may be closely positively related to the transcriptional abundance of $D 3$ gene in rice.

SLs are endogenous phytohormones that regulate diverse physiological processes, including shoot branching and elongation (Sang et al. 2014). D3 encodes an F-box containing protein, and is critical for SL signal transduction to suppress the shoot branching through assembled into an SCF complex and associated with D14 in rice (Zhao et al. 2014). Accumulating evidence shows that plant hormones have 
synergistic effects on each other. For instance, SL-deficient mutants la 1 and $d 3$ have increased IAA contents in both shoot base and whole seedlings, indicating that SL regulates the tiller/branch angle by inhibiting auxin biosynthesis in rice (Sang et al. 2014). The SL-insensitive (d14) and SL-deficient mutants (d17) are dwarf, and have reduced bioactive $\mathrm{GA}_{1}$ contents compared with WT, which could be rescued by application of exogenous $\mathrm{GA}_{3}$ (Zou et al. 2019). Accordingly, we speculate that D3 should be involved in multiple regulatory pathways in response to different plant hormones. In this study, the $d 3$ mutants have lower GA content and higher ABA content, which is more sensitive to GA but not to ABA; The dwarfness of $d 3$ mutants could be rescued by exogenous $\mathrm{GA}_{3}$ supplementation (Fig. 6). By contrast, OE-D3 seedlings have increased $G A$ content and restored $\mathrm{GA}_{3}$ and $A B A$ sensitivity like WT plants (Fig. 6). In view of the antagonizing regulation of $\mathrm{GA}$ and $\mathrm{ABA}$ in plants, it is reasonable to infer that miR528-D3 regulates shoot elongation by regulating the expression of the GA and ABA metabolic pathway-related genes (Fig. 7). Therefore, $D 3$, as a key regulatory node, may control plant height by mediating the signaling and metabolism of $\mathrm{SL}, \mathrm{GA}$, and $\mathrm{ABA}$ phytohormones in rice.

\section{Conclusion}

In the present study, the functional role of the miR528-D3 module in regulation of plant height in rice was investigated. It was found that miR528 and its targeting gene $D 3$ were oppositely expressed in tested tissues along developmental stages, where miR528 was mainly expressed in roots and leaves at the maturity stage. The plant height of $d 3$ and OE-miR528 lines was significantly lower than WT, OE-D3, and OE-MIM528 lines, with the former transgenics having severely shortened internodes. However, both WT and transgenics had the same number of internodes, while the cell length of internodes of OE-D3 and OEMIM528 lines was extensively longer than those of $d 3$ and OE-miR528 plants that contained apparently lower level of $\mathrm{GA}_{3}$ but higher $\mathrm{ABA}$ content. The supplementation of exogenous $\mathrm{GA}(1 \mu \mathrm{M})$ and $\mathrm{ABA}(3 \mu \mathrm{M})$ promoted the increase of plant height of $d 3$ and OE-miR528 lines, indicating that these transgenic lines were much more sensitive to external GA but not to ABA. Furthermore, GA biosynthesis-related genes (OsCPS1, OsCPS2, OsKO2 and OsKAO) were strongly expressed in OE-D3 plants, while ABA biosynthesisrelated gene $N E C D 2$ was highly expressed in $d 3$ mutant. The results are useful for further understanding and manipulating the regulatory mechanism of plant height in rice.

\section{Materials And Methods}

\section{Plant materials}

Oryza sativa L. japonica cv. Nipponbare was used as the wild type (WT) and genetic transformation in this study. The miR528 overexpressing plants (OE-miR528) and the miR528 target mimicry lines (OEMIM528) were developed in our previous studies (Liu et al. 2015; Wang et al. 2021). The OE-miR528, OEMIM528, OE-D3 lines, and $d 3$ mutants were created with the Nipponbare background and used in the following experiments.

\section{Generation of transgenic rice plants}


Using the full-length cDNA from the cv. Nipponbare as the template, the coding sequence (CDS) of the D3 gene (LOC_Os06g06050) was amplified using the specific primers listed in Table S1. The purified PCR product was then cloned into the $P C A M B I A 1300-U B I-R B C S$ vector using the Hieff Clone $\circledast$ Plus One Step Cloning Kit. The $D 3$ knockout vector was constructed using the CRISPR/Cas9 method. Based on the genomic sequence of $D 3$ gene, two specific CRISPR target sites were designed in the protein-encodable region. After determining the sites to be knocked out, the 23-25 bp exon sequence was selected, and subjected to obtain the sgRNA target site sequence for the specifically edited $D 3$ gene by using the Guide Design Resources (http://crispr.mit.edu/). Th gRNA vector was subsequently cut with Bsal and connected to the small fragment annealed at the target site. Then PCR amplification was performed to obtain the gRNA expression box containing the target site. After that, the recovered and amplified target site fragments were digested with $B s a l$, and multi-fragmented with the Cas9 vector digested with $B s a l$. The constructed CRISPR vector was then transferred into E. coli competent cells for proliferation. The constructs were separately transferred into Nipponbare by Agrobacterium-mediated transformation method to generate transgenic lines (Tzfira and Citovsky 2006).

The genomic DNA was extracted from the leaves of transgenic seedlings using the CTAB method, and used for positive identification. For knockout transgenic plants, the target site-specific primers were used for PCR amplification and sequencing to detect the editing sites (Table S1).

\section{Quantitative real-time PCR (qRT-PCR)}

Total RNA was extracted from different tissues using TRIzol reagent (TaKaRa). After treated with DNase I, the RNA was used to synthesize cDNA using the Hifair ${ }^{\text {rm }}$ III 1st Strand cDNA Synthesis Kit (YEASEN, China, http://www.yeasen.com). qRT-PCR was conducted in a BIO-RAD CFX96 Real-Time PCR System using the Hieff® qPCR SYBR® Green Master Mix (YEASEN, China, http://www.yeasen.com), according to the manufacturer's instructions. The total volume of the reaction system was $20 \mu \mathrm{L}$, including Hieff ${ }^{\circledR}$ qPCR SYBR Green Master Mix, $10 \mu \mathrm{L}$, Forward primer, $0.4 \mu \mathrm{L}$, Reverse primer, $0.4 \mu \mathrm{L}$, Template of cDNA, 9 $\mu \mathrm{L}$, and RNase-free water, $0.2 \mu \mathrm{L}$. The reaction procedure for qRT-PCR was as follows: $95^{\circ} \mathrm{C}$ for $5 \mathrm{~min}$, followed by 40 cycles of $95^{\circ} \mathrm{C}$ for $10 \mathrm{~s}, 55-60^{\circ} \mathrm{C}$ for $20 \mathrm{~s}$, and $72^{\circ} \mathrm{C}$ for $20 \mathrm{~s}$. All experiments were performed with three biological repeats and three technical replicates. The Ubquitin gene was used as the internal control for normalization. The $2^{-\triangle \Delta C T}$ method was adopted to evaluate the relative expression level of corresponding genes. Primers used for qRT-PCR were listed in Table S2.

\section{Histological analysis of cell morphology}

To explore the cell morphology of WT and transgenic plants, the same region of the uppermost internode was collected from 31-day-old seedlings, and fixed in $75 \%$ alcohol solution immediately. The rice samples were heated in a water bath until the leaves turned into white completely, and then oven-dried at $37^{\circ} \mathrm{C}$ overnight. The samples were put under an optical microscope to observe the cell morphology and count cell numbers. The cell length of the uppermost internode was measured using ImageJ (Collins 2007). The measurement was conducted for more than 15 cells each of three plants per line.

\section{Determination of endogenous $\mathrm{GA}$ and $\mathrm{ABA}$ content}


For GA and ABA quantification, $1.5 \mathrm{~g}$ of stems of 31-day-old WT and transgenic rice plants were collected and powdered with liquid nitrogen. Each sample was extracted in $80 \%$ methanol (with $1 \mathrm{mM}$ 2-tert-Butyl4-methylphenol), and centrifuged at $4000 \mathrm{rpm}$ for $8 \mathrm{~min}$ at $4^{\circ} \mathrm{C}$. The extract was blow-dried with nitrogen and dissolved in phosphate buffer ( $\mathrm{pH}$ 7.5). The total concentrations of $\mathrm{GA}_{3}$ and $\mathrm{ABA}$ were determined by enzyme-linked immunoassay (ELISA), as described previously (Luo et al. 2006). Each assay was performed with three biological replicates.

\section{Exogenous GA and ABA treatment}

Rice seeds were sterilized with $0.5 \% \mathrm{NaClO}$ solution for $15 \mathrm{~min}$, and washed three times with sterile distilled water, and then immersed in water for two days in a constant temperature incubator at $32^{\circ} \mathrm{C}$. Germinated seeds were grown on a nylon net floating on hydroponic solution (pH 5.8) (Shim et al. 2021). The 14-day-old seedlings of WT and transgenic lines were treated with $1 \mu \mathrm{M} \mathrm{GA}_{3}$ or $3 \mu \mathrm{M} \mathrm{ABA}$ for two weeks. Each treatment experiment was performed with three replicates with twelve seedlings. The nutrient solution was renewed every three days. After the 7 and $14 \mathrm{~d}$ of hormone treatment, the shoot length of each plant was measured (Chu et al. 2019; Chen et al. 2021).

\section{Abbreviations}

ABA: Abscisic acid; BR: Brassinosteroids; D3: Dwarf 3; ELISA: Enzyme-linked immunoassay; GA:

Gibberellin; IM: Intermediate meristem; miRNA: microRNA; SL: Strigolactone; SAM: Shoot apical meristem; WT: Wild type

\section{Declarations}

\section{Ethics Approval and Consent to Participate}

Not applicable.

\section{Consent for Publication}

Not applicable.

\section{Availability of Supporting Data}

The datasets supporting the conclusions of this article are included within the article and its additional files.

\section{Competing Interests}

The authors declare that they have no competing interests.

\section{Funding}


This work was financially supported by grants from the National Natural Science Foundation of China (31972959) and the Key Project of Zhejiang Provincial Natural Science Foundation of China (LZ19B070001) to Q.L.

\section{Authors' Contributions}

$Q \mathrm{~L}$ conceived and designed the experiment, and supervised the project. $\mathrm{Q} L, X L$ and $\mathrm{J} Z$ wrote the manuscript. $X L, M W, J Z, L X, Z W, J Y, Y W, Z Z$ and $Y J$ performed the experiments and analyzed the data. All authors read and approved the final manuscript.

\section{Acknowledgments}

Not applicable.

\section{References}

1. Arite T, Umehara M, Ishikawa S, Hanada A, Maekawa M, Yamaguchi S, Kyozuka J (2009) d14, a strigolactone-insensitive mutant of rice, shows an accelerated outgrowth of tillers. Plant Cell Physiology 50:1416-1424

2. Ayano M, Kani T, Kojima M, Sakakibara H, Ashikari M (2015) Gibberellin biosynthesis and signal transduction is essential for internode elongation in deepwater rice. Plant Cell Environment 37:23132324

3. Bang SW, Park SH, Jeong SJ, Kim YS, Jung H, Ha SH, Kim JK (2013) Characterization of the stressinducible OSNCED3 promoter in different transgenic rice organs and over three homozygous generations. Planta 237:211-224

4. Chen Y, Shen J, Zhang L, Qi H, Yang L, Wang H, Wang J, Wang Y, Du H, Tao Z, Zhao T, Deng P, Shu Q, Qian Q, Yu H, Song S (2021) Nuclear translocation of OsMFT1 that is impeded by OsFTIP1 promotes drought tolerance in rice. Mol Plant 14:1297-1311

5. Chi S, Mei Z, Duan J, Chen H, Feng H, Cai W, Gloria M (2014) OsGA2ox5, a gibberellin metabolism enzyme, is involved in plant growth, the root gravity response and salt stress. PLoS One 9:e87110

6. Choi J, Lee T, Cho J, Servante EK, Pucker B, Summers W, Bowden S, Rahimi M, An K, An G, Bouwmeester HJ, Wallington EJ, Oldroyd G, Paszkowski U (2020) The negative regulator SMAX1 controls mycorrhizal symbiosis and strigolactone biosynthesis in rice. Nat Commun 11:2114

7. Chu Y, Xu N, Wu Q, Yu B, Li X, Chen R, Huang J (2019) Rice transcription factor OsMADS57 regulates plant height by modulating gibberellin catabolism. Rice 12:38

8. Collins TJ (2007) ImageJ for microscopy. Biotechniques 43:25-30

9. Endo A, Nelson KM, Thoms K, Abrams SR, Nambara E, Sato Y (2014) Functional characterization of xanthoxin dehydrogenase in rice. J Plant Physiol 171:1231-1240

10. Feng Q, Zhao KJ (2011) The influence of RNAi targeting of OsGA20ox2 gene on plant height in rice. Plant Molecular Biology Reporter 29:952 
11. Gao Z, Qian Q, Liu X, Yan M, Feng Q, Dong G, Liu J, Han B (2009) Dwarf 88, a novel putative esterase gene affecting architecture of rice plant. Plant Mol Biol 71:265-276

12. Hong Z, Ueguchi-Tanaka M, Fujioka S, Takatsuto S, Yoshida S, Hasegawa Y, Ashikari M, Kitano H, Matsuoka M (2005) The rice brassinosteroid-deficient dwarf2 mutant, defective in the rice homolog of Arabidopsis DIMINUTO/DWARF1, is rescued by the endogenously accumulated alternative bioactive brassinosteroid, dolichosterone. Plant Cell 17:2243-2254

13. Huang J, Tang D, Shen Y, Qin B, Hong L, You A, Li M, Wang X, Yu H, Gu M, Cheng Z (2010) Activation of gibberellin 2-oxidase 6 decreases active gibberellin levels and creates a dominant semi-dwarf phenotype in rice (Oryza sativa L.). Journal of Genetics Genomics 37:23-36

14. Ishikawa S, Maekawa M, Arite T, Onishi K, Takamure I, Kyozuka J (2005) Suppression of tiller bud activity in tillering dwarf mutants of rice. Plant Cell Physiology 46:79-86

15. Itoh H, Tatsumi T, Sakamoto T, Otomo K, Toyomasu T, Kitano H, Ashikari M, Ichihara S, Matsuoka M (2004) A rice semi-dwarf gene, Tan-Ginbozu (D35), encodes the gibberellin biosynthesis enzyme, entkaurene oxidase. Plant Molecular Biology 54: 533-547

16. Li YF, Zheng Y, Addo-Quaye C, Zhang L, Saini A, Jagadeeswaran G, Axtell MJ, Zhang W, Sunkar R (2010) Transcriptome-wide identification of microRNA targets in rice. Plant J 62:742-759

17. Lin H, Wang R, Qian Q, Yan M, Meng X, Fu Z, Yan C, Jiang B, Su Z, Li J, Wang Y (2009) DWARF27, an iron-containing protein required for the biosynthesis of strigolactones, regulates rice tiller bud outgrowth. Plant Cell 21:1512-1525

18. Liu Q, Hu H, Zhu L, Li R, Feng Y, Zhang L, Yang Y, Liu X, Zhang H (2015) Involvement of miR528 in the regulation of arsenite tolerance in rice (Oryza sativa L.). J Agric Food Chem 63:8849-8861

19. Lo SF, Yang SY, Chen KT, Hsing YI, Zeevaart JAD, Chen LJ, Yu SM (2008) A novel class of gibberellin 2-oxidases control semidwarfism, tillering, and root development in rice. Plant Cell 20:2603-2618

20. Luo A, Qian Q, Yin H, Liu X, Yin C, Lan Y, Tang J, Tang Z, Cao S, Wang X, Xia K, Fu X, Luo D, Chu C (2006) EUI1, encoding a putative cytochrome P450 monooxygenase, regulates internode elongation by modulating gibberellin responses in rice. Plant Cell Physiology 47:181-191

21. Monna L, Kitazawa N, Yoshino R, Suzuki J, Masuda H, Maehara Y, Tanji M, Sato M, Nasu S, Minobe Y (2002) Positional cloning of rice semidwarfing gene, sd-1: rice "green revolution gene" encodes a mutant enzyme involved in gibberellin synthesis. DNA Res 9:11-17

22. Oikawa T, Koshioka M, Kojima K, Yoshida H, Kawata M (2004) A role of OsGA20ox1, encoding an isoform of gibberellin 20-oxidase, for regulation of plant stature in rice. Plant Mol Biol 55:687-700

23. Qin X, Liu JH, Zhao WS, Chen XJ, Guo ZJ, Peng YL (2013) Gibberellin 20-oxidase gene OsGA20ox3 regulates plant stature and disease development in rice. Molecular Plant Microbe Interactions 26:227-239

24. Saika H, Okamoto M, Miyoshi K, Kushiro T, Shinoda S, Jikumaru Y, Fujimoto M, Arikawa T, Takahashi $\mathrm{H}$, Ando M, Arimura SI, Miyao A, Hirochika H, Kamiya Y, Tsutsumi N, Nambara E, Nakazono M (2007) Ethylene promotes submergence-induced expression of OSABA80xl, a gene that encodes ABA 8'hydroxylase in rice. Plant Cell Physiology 48:287-298

Page $11 / 19$ 
25. Sakamoto T, Miura K, Itoh H, Tatsumi T, Ueguchi-Tanaka M, Ishiyama K, Kobayashi M, Agrawal G, Takeda S, Abe K, Miyao A, Hirochika H, Kitano H, Ashikari M, Matsuoka M (2004) An overview of gibberellin metabolism enzyme genes and their related mutants in rice. Plant Physiol 134:16421653

26. Sang D, Chen D, Liu G, Liang Y, Huang L, Meng X, Chu J, Sun X, Dong G, Yuan Y, Qian Q, Li J, Wang Y (2014) Strigolactones regulate rice tiller angle by attenuating shoot gravitropism through inhibiting auxin biosynthesis. Procedings of the National Academy of Sciences of the United States of America 111: $11199-11204$

27. Shim JS, Park SH, Lee DK, Kim YS, Park SC, Redillas MCFR, Seo JS, Kim JK (2021) The rice GLYCINERICH PROTEIN 3 confers drought tolerance by regulating mRNA stability of ROS scavenging-related genes. Rice 14:31

28. Spielmeyer W, Ellis MH, Chandler PM (2002) Semidwarf (sd-1), "green revolution" rice, contains a defective gibberellin 20-oxidase gene. Proc Natl Acad Sci USA 99:9043-9048

29. Sun S, Wang T, Wang L, Li X, Jia Y, Liu C, Huang X, Xie W, Wang X (2018) Natural selection of a GSK3 determines rice mesocotyl domestication by coordinating strigolactone and brassinosteroid signaling. Nat Commun 9:2523

30. Tang W, Thompson WA (2019) OsmiR528 enhances cold stress tolerance by repressing expression of stress response-related transcription factor genes in plant cells. Curr Genomics 20:100-114

31. Tong H, Xiao Y, Liu D, Gao S, Liu L, Yin Y, Jin Y, Qian Q, Chu C (2014) Brassinosteroid regulates cell elongation by modulating gibberellin metabolism in rice. Plant Cell 26:4376-4393

32. Tong X, Wang Y, Sun A, Bello BK, Ni S, Zhang J (2018) Notched belly grain 4, a novel allele of dwarf 11, regulates grain shape and seed germination in rice (Oryza sativa L.). International Journal of Molecular Science 19:4069

33. Toyomasu T, Kagahara T, Hirose Y, Usui M, Abe S, OKADA K, Koga J, Mitsuhashi W, Yamane H (2009) Cloning and characterization of cDNAs encoding ent-copalyl diphosphate synthases in wheat: insight into the evolution of rice phytoalexin biosynthetic genes. Bioscience Biotechnology Biochemistry 73:772-775

34. Tzfira T, Citovsky V (2006) Agrobacterium-mediated genetic transformation of plants: biology and biotechnology. Curr Opin Biotechnol 17:147-154

35. Ueguchi-Tanaka M, Ashikari M, Nakajima M, Itoh H, Katoh E, Kobayashi M, Chow TY, Hsing YI, Kitano H, Yamaguchi I, Matsuoka M (2005) GIBBERELLIN INSENSITIVE DWARF1 encodes a soluble receptor for gibberellin. Nature 437:693-698

36. Wang B, Smith SM, Li J (2018) Genetic regulation of shoot architecture. Annu Rev Plant Biol 69:437468

37. Wang B, Wang H (2017) IPA1: a new "green revolution". gene? Molecular Plant 10:779-781

38. Wang M, Guo W, Li J, Pan X, Pan L, Zhao J, Zhang Y, Cai S, Huang X, Wang A, Liu Q (2021) The miR528- $A O$ module confers enhanced salt tolerance in rice by modulating the ascorbic acid and abscisic acid metabolism and ROS scavenging. J Agric Food Chem. DOI:10.1021/acs.jafc.1c01096 
39. Wang Y, Hou Y, Qiu J, Wang H, Wang S, Tang L, Tong X, Zhang J (2020) Abscisic acid promotes jasmonic acid biosynthesis via a 'SAPK10-bZIP72-AOC' pathway to synergistically inhibit seed germination in rice (Oryza sativa). New Phytol 228:1336-1353

40. Wu J, Yang R, Yang Z, Yao S, Zhao S, Wang Y, Li P, Song X, Jin L, Zhou T, Lan Y, Xie L, Zhou X, Chu C, Qi Y, Cao X, Li Y (2017) ROS accumulation and antiviral defence control by microRNA528 in rice. Nature Plants 3:16203

41. Wu J, Zhu C, Pang J, Zhang X, Yang C, Xia G, Tian Y, He C (2014) OsLOL1, a C2C2-type zinc finger protein, interacts with OsbZIP58 to promote seed germination through the modulation of gibberellin biosynthesis in Oryza sativa. Plant J 80:1118-1130

42. Yan H, Saika H, Maekawa M, Takamure I, Tsutsumi N, Kyozuka J, Nakazono M (2007) Rice tillering dwarf mutant dwarf3 has increased leaf longevity during darkness-induced senescence or hydrogen peroxide-induced cell death. Genes Genet Syst 82:361-366

43. Yang M, Qi W, Sun F, Zha X, Chen M, Huang Y, Feng YQ, Yang J, Luo X (2013) Overexpression of rice LRK1 restricts internode elongation by down-regulating OsKO2. Biotech Lett 35:121-128

44. Yang R, Li P, Mei H, Wang D, Sun J, Yang C, Hao L, Cao S, Chu C, Hu S, Song X, Cao X (2019) Finetuning of miR528 accumulation modulates flowering time in rice. Mol Plant 12:1103-1113

45. Zhang B, Wang $X$, Zhao Z, Wang R, Huang X, Zhu Y, Yuan L, Wang Y, Xu X, Burlingame AL, Gao Y, Sun $Y$, Tang W (2016) OsBRI1 activates BR signaling by preventing binding between the TPR and kinase domains of OsBSK3 via phosphorylation. Plant Physiol 170:1149-1161

46. Zhang J, Zhang X, Chen R, Yang L, Fan K, Liu Y, Wang G, Ren Z, Liu Y (2020a) Generation of transgene-free semidwarf maize plants by gene editing of gibberellin-oxidase20-3 using CRISPR/Cas9. Front Plant Sci 11:1048

47. Zhang Y, Wang X, Luo Y, Zhang L, Yao Y, Han L, Chen Z, Wang L, Li Y (2020c) OsABA8ox2, an ABA catabolic gene, suppresses root elongation of rice seedlings and contributes to drought response. The Crop Journal 8:480-491

48. Zhang Y, Zhang S, Jing L, Wang Y, Fang X (2014) Research progress on cloning and functional analysis of plant height genes in rice (Oryza sativa L.). Chin Agric Sci Bull 12:1-7

49. Zhang Y, Zhu Y, Peng Y, Yan D, Li Q, Wang J, Wang L, He Z (2008) Gibberellin homeostasis and plant height control by EUI and a role for gibberellin in root gravity responses in rice. Cell Res 18:412-421

50. Zhang YC, He RR, Lian JP, Zhou YF, Zhang F, Li QF, Yu Y, Feng YZ, Yang YW, Lei MQ, He H, Zhang Z, Chen YQ (2020b) OsmiR528 regulates rice-pollen intine formation by targeting an uclacyanin to influence flavonoid metabolism. Proc Natl Acad Sci USA 117:727-732

51. Zhao J, Wang T, Wang M, Liu Y, Yuan S, Gao Y, Yin L, Sun W, Peng L, Zhang W, Wan J, Li X (2014) DWARF3 participates in an SCF complex and associates with DWARF14 to suppress rice shoot branching. Plant Cell Physiology 55:1096-1109

52. Zheng J, Hong K, Zeng L, Wang L, Kang S, Qu M, Dai J, Zou L, Zhu L, Tang Z, Meng X, Wang B, Hu J, Zeng D, Zhao Y, Cui P, Wang Q, Qian Q, Wang Y, Li J, Xiong G (2020) Karrikin signaling acts parallel to 
and additively with strigolactone signaling to regulate rice mesocotyl elongation in darkness. Plant Cell 32:2780-2805

53. Zhou M, Gu L, Li P, Song X, Wei L, Chen Z, Cao X (2010) Degradome sequencing reveals endogenous small RNA targets in rice (Oryza sativa L. ssp. indica). Frontiers in Biology 5: 67-90

54. Zhu G, Ye N, Zhang J (2009) Glucose-induced delay of seed germination in rice is mediated by the suppression of ABA catabolism rather than an enhancement of ABA biosynthesis. Plant Cell Physiology 50:644-651

55. Zou X, Wang Q, Chen P, Yin C, Lin Y (2019) Strigolactones regulate shoot elongation by mediating gibberellin metabolism and signaling in rice (Oryza sativa L.). J Plant Physiol 237:72-79

\section{Figures}
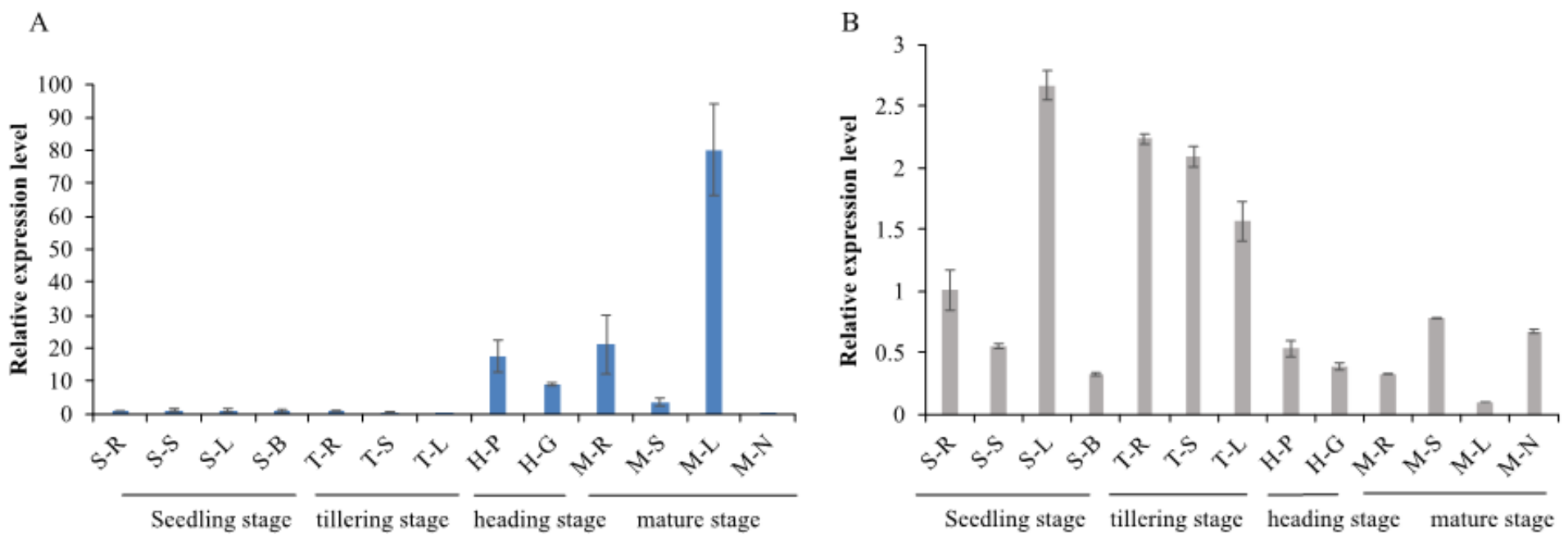

\section{Figure 1}

Analysis of the miR528 and D3 genes expression pattern in rice. (A) Expression pattern analysis of miR528 in various tissues by qRT-PCR. (B) Expression pattern analysis of D3 in various tissues by qRTPCR. S-R, root at the 7-day seedlings; S-S, stem at the 7-day seedlings; S-L, leaf at the 7-day seedlings; S-B, stem base at the 7-day seedlings; T-R, root at the tillering stage; T-S, stem at the tillering stage; T-L leaf at the tillering stage; $\mathrm{H}-\mathrm{P}$, Panicles at the heading stage; $\mathrm{H}-\mathrm{G}$, glume at the heading stage; $\mathrm{M}-\mathrm{R}$, root at the mature stage; $M-S$, stem at the mature stage; $M-L$, leaf at the mature stage; $M-N$, stem node at the mature stage. Data are shown as the means \pm S.D. $(n=3)$. 

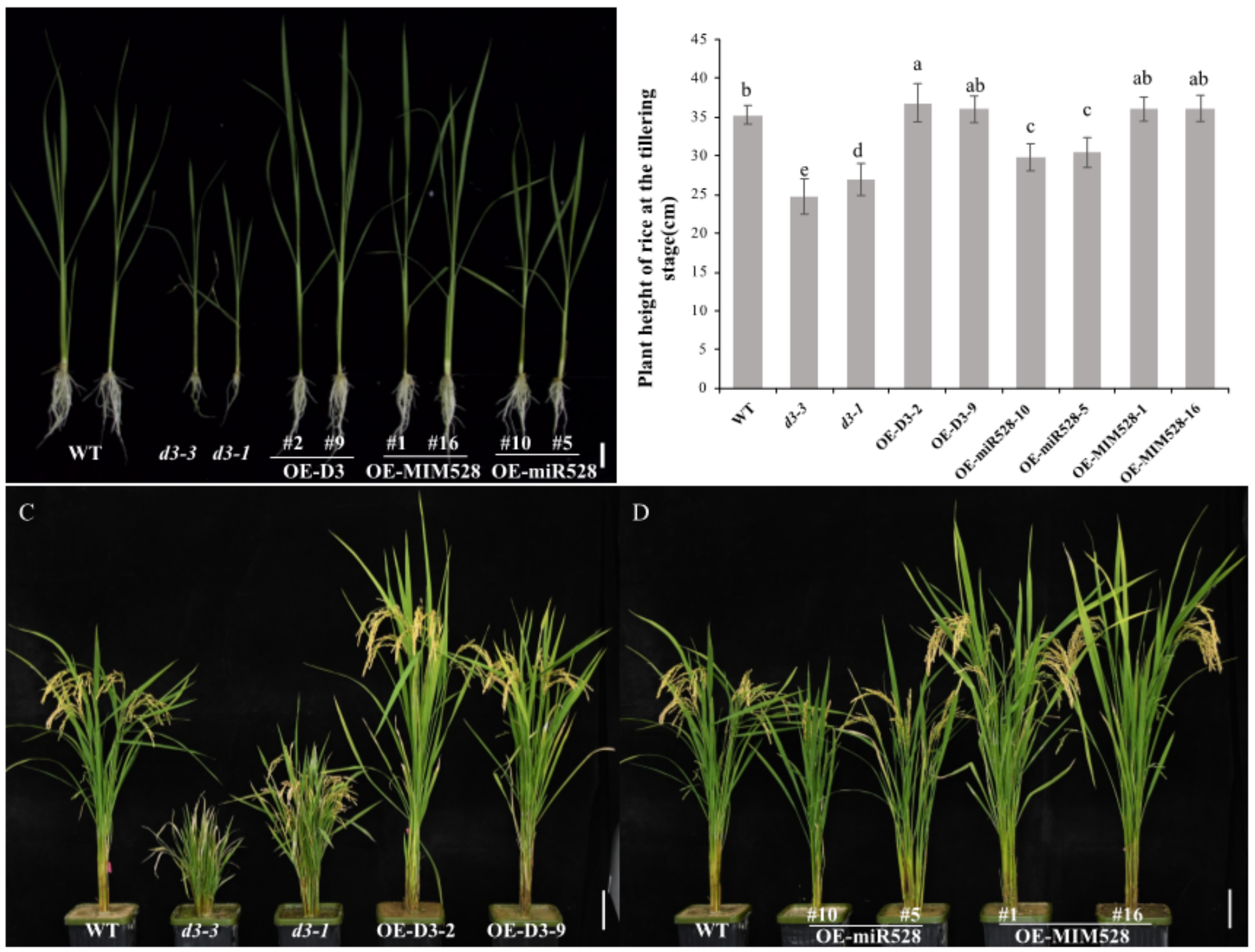

Figure 2

Comparison of plant height between WT and transgenic lines at the tillering and maturity stages. (A) Phenotypes of WT and transgenic lines at the tillering stage. Bars, $2 \mathrm{~cm}$. (B) Comparison of plant height at the tillering stage. Data are shown as the means \pm S.D. $(n=10)$. Significant differences $(P<0.05)$ are indicated by different letters. (C-D) Phenotypes of WT and transgenic lines at the maturity stage. Bars, 10 $\mathrm{cm}$. Data are shown as the means \pm S.D. $(n=10)$. 

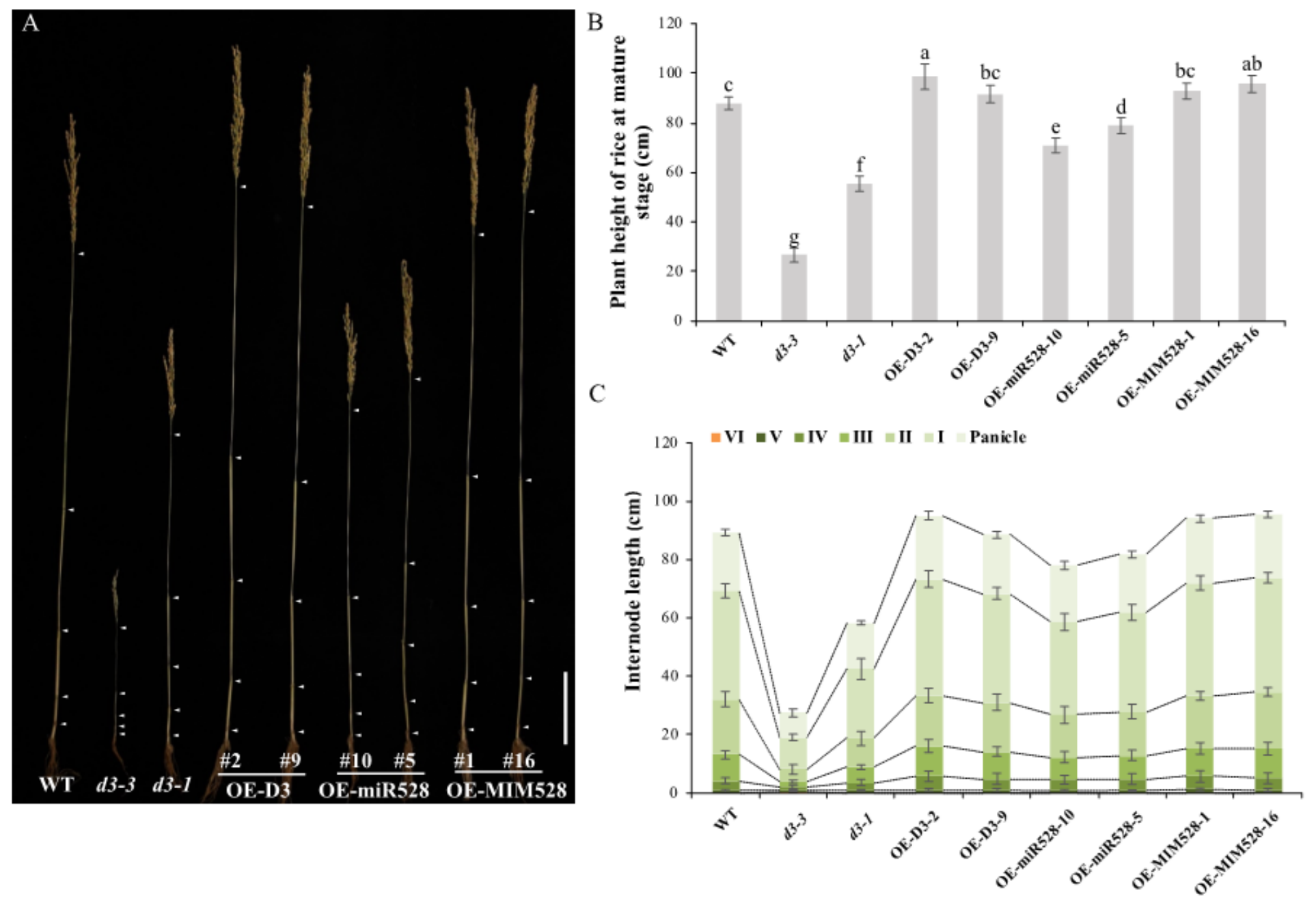

Figure 3

Comparison of the length of internodes of stems between WT and transgenic lines. (A) Internode morphology of WT and transgenic lines. Bars, $10 \mathrm{~cm}$. (B) Comparison of plant height at the maturity stage. Data are shown as the means \pm S.D. $(n=10)$. Significant differences $(P<0.05)$ are indicated by different letters. (C) Comparison of internode length of WT and transgenic lines. Data are shown as the means \pm S.D. $(n=10)$. 

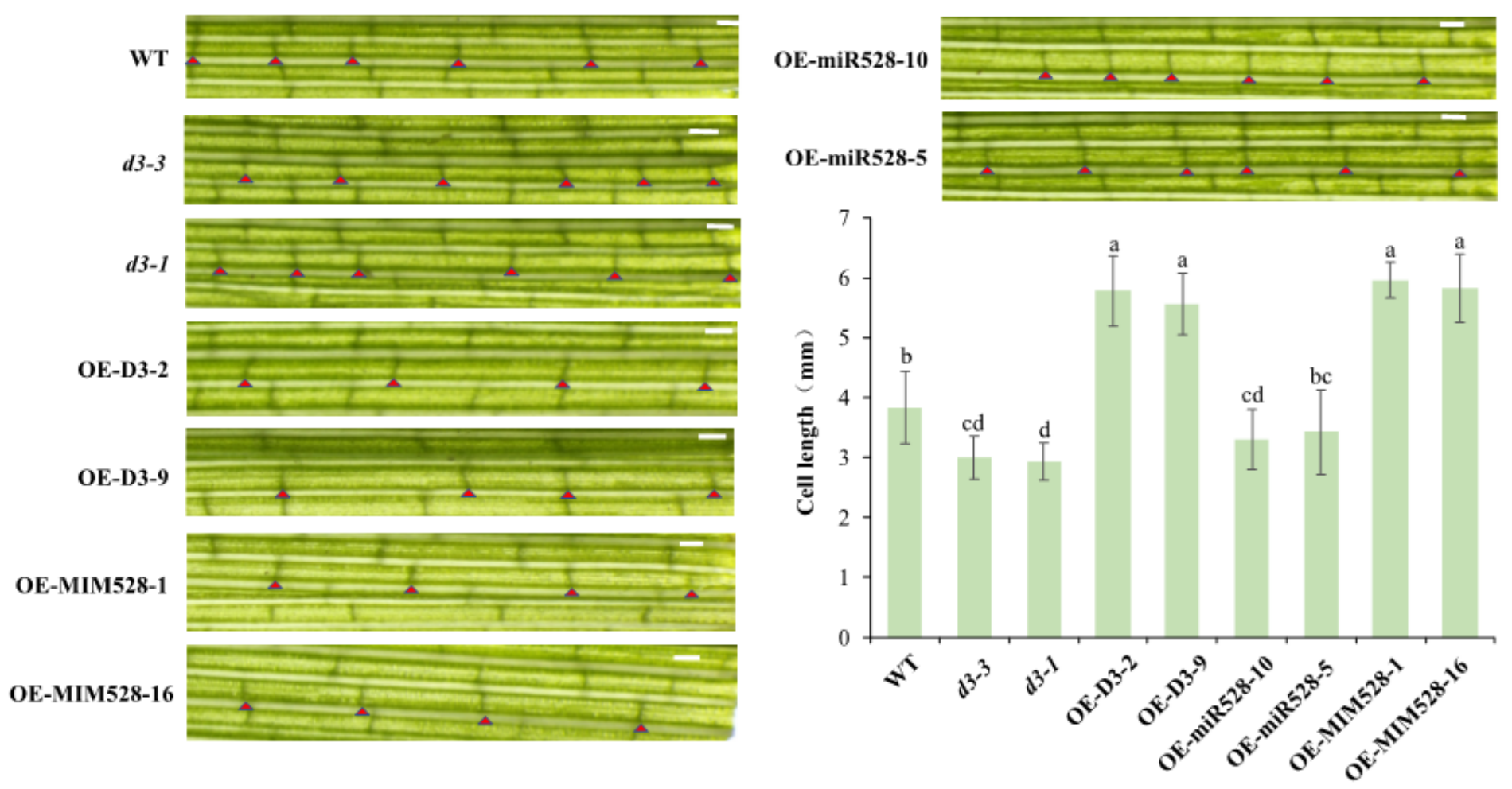

\section{Figure 4}

Phenotypic analysis of the internode length of WT and transgenic lines. Longitudinal section of the uppermost internode from WT and transgenic lines at the tillering stage, and statistical analysis of the cell length of internodes in WT and transgenic plants. Data are shown as the means \pm S.D. $(n=10)$. Bars, $1 \mathrm{~mm}$. Significant differences $(P<0.05)$ are indicated by different letters.
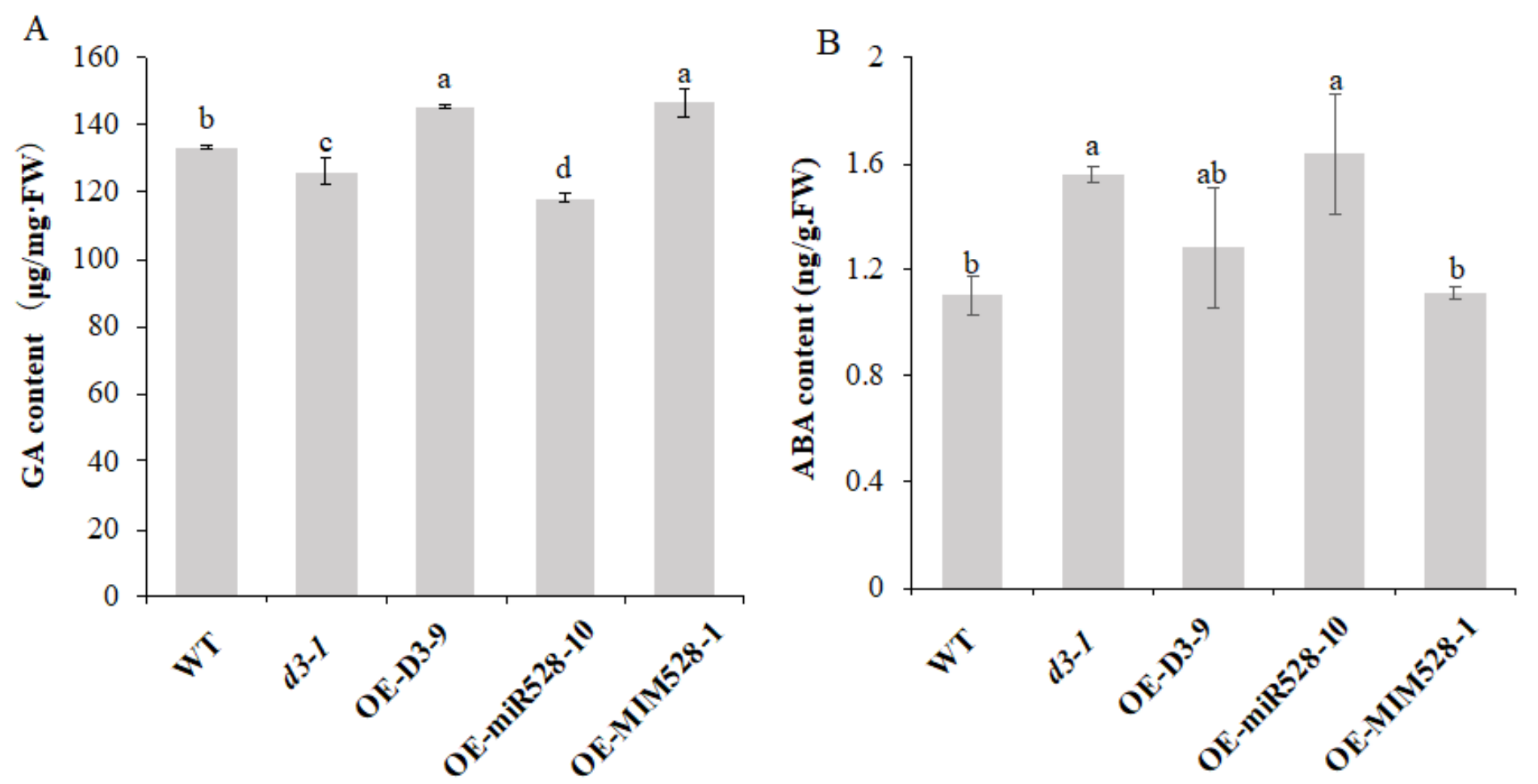


\section{Figure 5}

Comparative analysis of endogenous GA3 (A) and ABA content (B) in shoots of 31-day-old seedlings of WT and transgenic lines. Significant differences $(P<0.05)$ are indicated by different letters.

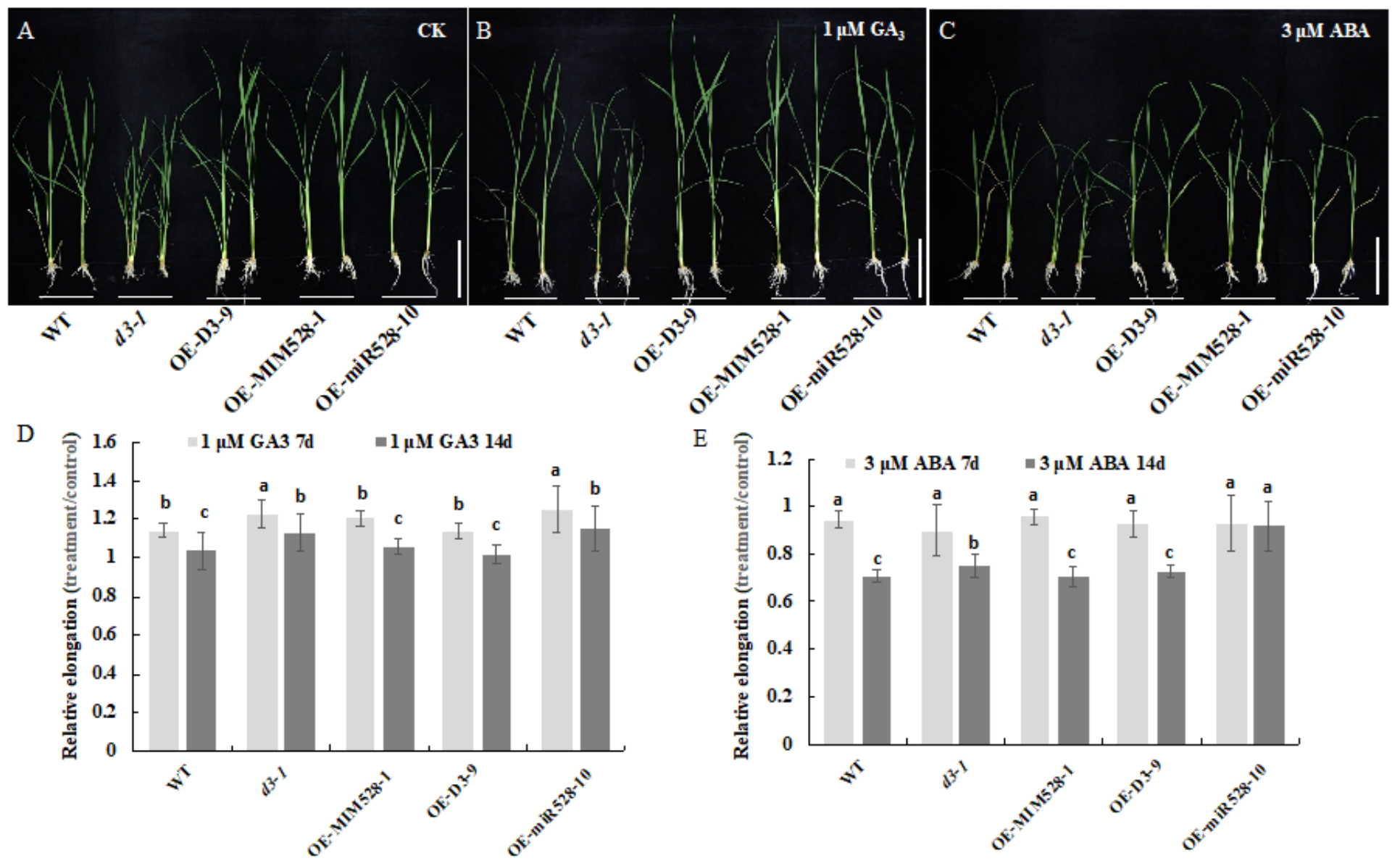

\section{Figure 6}

Response of WT and transgenic lines to exogenous GA and ABA treatment. (A) Phenotype of WT and transgenic plants under normal conditions; (B-C) Phenotypes of 14-day-old seedlings of WT and transgenic lines under the treatment with exogenous $1 \mu \mathrm{M}$ GA3 (B) and $3 \mu \mathrm{M} A B A$ (C) for 7 days. (D) Relative elongation of plant height after $1 \mu \mathrm{M}$ GA3 treatment for 7 and 14 days. (E) Relative elongation of plant height after $3 \mu \mathrm{M}$ ABA treatment for 7 and 14 days. Data are shown as the means \pm S.D. $(n=10)$. Significant differences $(P<0.05)$ are indicated by different letters. 


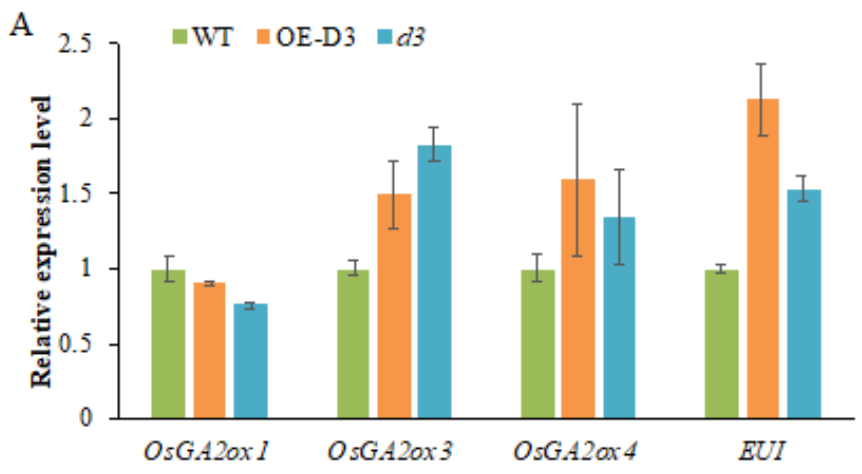

B
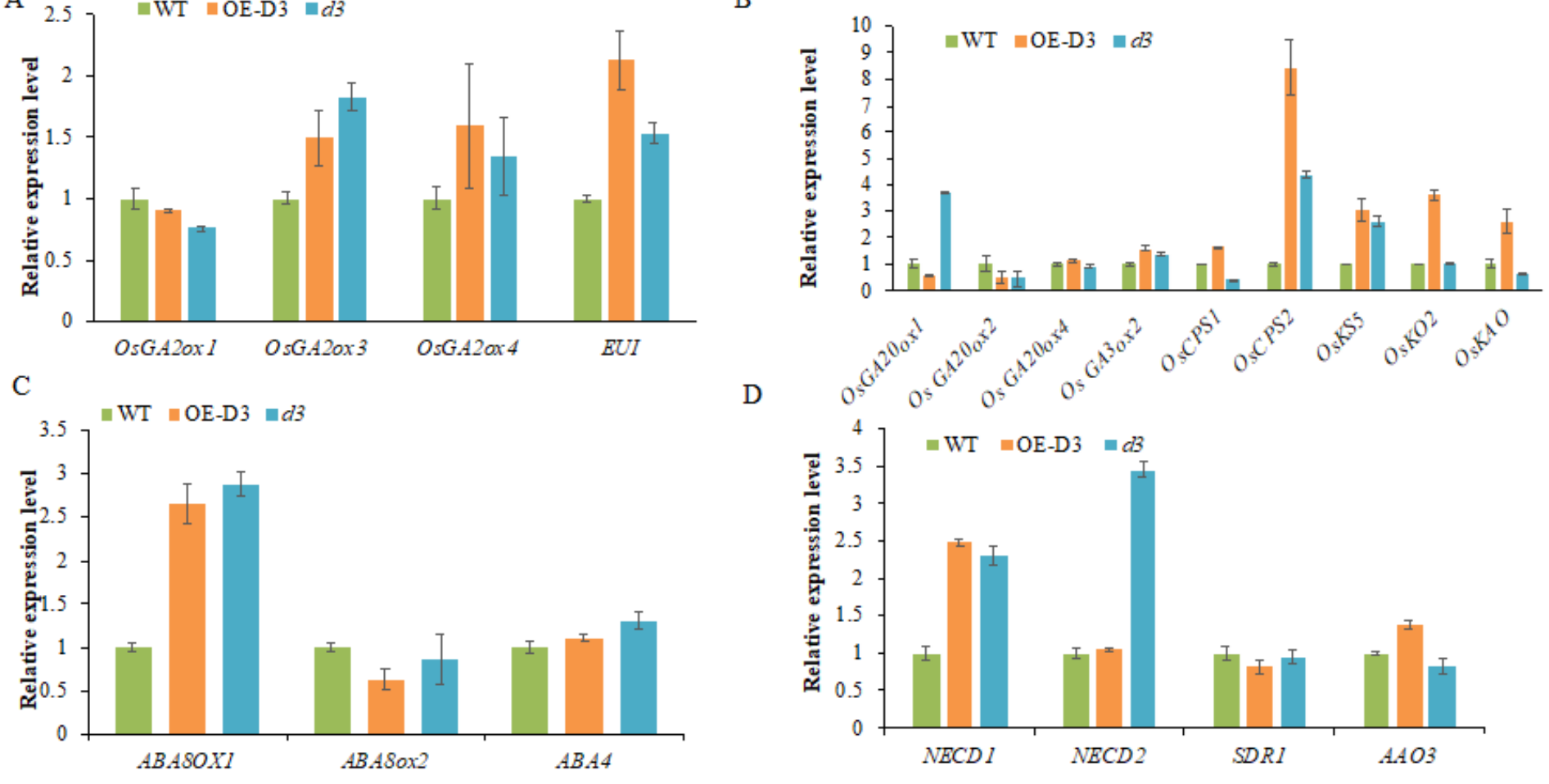

Figure 7

Expression analysis of some key genes involved in GA and ABA degradation and biosynthesis in WT, OED3 and d3 lines. (A) Expression analysis of GA degradation-related genes. (B) Expression analysis of GA biosynthesis-related genes. (C) Expression analysis of ABA degradation-related genes. (D) Expression analysis of $A B A$ biosynthesis-related genes. Data are presented as the means \pm S.D. of three biological replicates. The Ubquitin gene was used as an internal control. The relative expression level was calculated by the $2-\Delta \triangle C T$ method.

\section{Supplementary Files}

This is a list of supplementary files associated with this preprint. Click to download.

- 2supplementarymaterial20210817.pdf 